\title{
Carotid body inflammation and cardiorespiratory alterations in intermittent hypoxia
}

\author{
Rodrigo Del Rio, Esteban A. Moya, María J. Parga, \\ Carlos Madrid and Rodrigo Iturriaga
}

ABSTRACT: Chronic intermittent hypoxia $(\mathrm{ClH})$, a main feature of obstructive sleep apnoea (OSA), increases hypoxic ventilatory responses and elicits hypertension, partially attributed to an enhance carotid body (CB) responsiveness to hypoxia. As inflammation has been involved in $\mathrm{CIH}$ induced hypertension and chemosensory potentiation, we tested whether ibuprofen may block CB chemosensory and cardiorespiratory alterations induced by $\mathrm{CIH}$ in a rat model of OSA.

We studied the effects of ibuprofen $\left(40 \mathrm{mg} \cdot \mathrm{kg}^{-1} \cdot \mathrm{day}^{-1}\right)$ on immunohistochemical interleukin (IL)$1 \beta$ and tumour necrosis factor (TNF)- $\alpha$ levels in the $C B$, the number of $c$-fos-positive neurons in the nucleus tractus solitarii (NTS), CB chemosensory and ventilatory responses to hypoxia, and arterial blood pressure in male rats either exposed for 21 days to $5 \% \mathrm{O}_{2}\left(12\right.$ episodes $\cdot h^{-1}$, $8 \mathrm{~h} \cdot \mathrm{day}^{-1}$ ) or kept under sham condition.

$\mathrm{CIH}$ increased CB TNF- $\alpha$ and IL-1 $\beta$ and c-fos-positive neurons in the NTS, enhanced carotid chemosensory and ventilatory hypoxic responses, and produced hypertension. Ibuprofen prevented CB cytokine overexpression and $\mathrm{ClH}$-induced increases in c-fos-positive neurons in the NTS, the enhanced hypoxic ventilatory responses and hypertension, but failed to impede the CB chemosensory potentiation.

Results suggest that pro-inflammatory cytokines may contribute to the ClH-induced cardiorespiratory alterations, acting at several levels of the hypoxic chemoreflex and cardiovascular control pathways.

KEYWORDS: Hypertension, hypoxia, inflammation, obstructive sleep apnoea

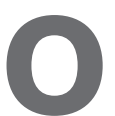
bstructive sleep apnoea (OSA) syndrome, a rising worldwide health problem, is characterised by chronic intermittent hypoxia $(\mathrm{CIH})$, which is considered the main risk factor for developing hypertension and other cardiovascular diseases [1-3]. It has been proposed that oxidative stress, inflammation and sympathetic activation are involved in OSA-induced hypertension [3-6]. A growing body of evidence suggests that $\mathrm{CIH}$ enhances carotid body (CB) chemosensory responses to hypoxia contributing to the OSA-induced hypertension [6-9]. Indeed, OSA patients and animals exposed to $\mathrm{CIH}$ show potentiated ventilatory, sympathetic and cardiovascular responses to acute hypoxia [6-10]. Furthermore, recordings of carotid chemosensory discharges in situ and in vitro have shown that $\mathrm{CIH}$ selectively increases basal chemosensory discharges in normoxia and potentiates the chemosensory responses to acute hypoxia in rats and cats $[9,11-13]$.

The repetitive episodes of hypoxia-reoxygenation during $\mathrm{CIH}$ exposure elicits oxidative stress due to the accumulation of reactive oxygen species (ROS), which are involved in the potentiation of the hypoxic $\mathrm{CB}$ chemosensory responses $[9,11,13,14]$ and in the pathological consequences observed in animals exposed to $\mathrm{CIH}$, and in OSA patients [3-5, 8, 9, 13]. Recently, we found that ascorbic acid supplementation, which impedes the systemic and local $\mathrm{CB}$ oxidative stress in the rat exposed to $\mathrm{CIH}$ for 21 days, prevented enhanced CB chemosensory and ventilatory responses to hypoxia, as well as hypertension [13]. These observations support a main contribution for oxidative stress in the generation of the CB chemosensory potentiation
AFFILIATIONS

Laboratorio de Neurobiología,

Facultad de Ciencias Biológicas, Pontificia Universidad Católica de Chile, Santiago, Chile.

CORRESPONDENCE

R. Iturriaga

Laboratorio de Neurobiología

Facultad de Ciencias Biológicas

Pontificia Universidad Católica de

Chile

Casilla 114-D

Santiago

Chile

E-mail: riturriaga@bio.puc.cl

Received:

Aug 172011

Accepted after revision:

Nov 042011

First published online:

Dec 192011 
and the cardiorespiratory alterations induced by $\mathrm{CIH}$. Nevertheless, a direct effect of ROS in the CB oxygen process is still matter for debate. Indeed, the application of $\mathrm{H}_{2} \mathrm{O}_{2}$ does not produce $\mathrm{CB}$ chemosensory excitation $[15,16]$. Moreover, modifications of ROS production in the CB do not modify the catecholaminergic secretory response to hypoxia, indicating a lack of a causal link between ROS levels and chemoreceptor activity [17]. Thus, it is likely that other molecules downstream of the ROS signals may mediate the enhancing effects of ROS on $\mathrm{CB}$ chemoreception under intermittent hypoxia. Among other molecules upregulated in the $\mathrm{CB}$ by $\mathrm{CIH}$, such as endothelin (ET)-1 and inductible nitric oxide synthase [14, 18, 19], pro-inflammatory cytokines have been proposed as mediators of the $\mathrm{CB}$ chemosensory potentiation induced by $\mathrm{CIH}[9,14]$. Indeed, recently we found that $\mathrm{CIH}$ for 21 days increases the expression of tumour necrosis factor (TNF)- $\alpha$ and interleukin (IL)-1 $\beta$ in rat CB [9], molecules which are considered excitatory modulators of the $\mathrm{CB}$ oxygen chemoreception $[20,21]$.

The progression of the hypertension in OSA patients and animals exposed to $\mathrm{CIH}$ is also associated with increased levels of pro-inflammatory cytokines [1, 3, 5]. An increased ROS production induced by hypoxia-reoxygenation evokes the synthesis and secretion of pro-inflammatory cytokines [22]. Thus, we hypothesised that a treatment with an anti-inflammatory drug may prevent both the $\mathrm{CB}$ chemosensory potentiation and the cardiorespiratory alterations in rats exposed to $\mathrm{CIH}$. Accordingly, we studied the effects of the nonsteroidal antiinflammatory drug ibuprofen on the increased immunoreactive levels of TNF- $\alpha$ and IL- $1 \beta$ in rat CB, the potentiation of the $\mathrm{CB}$ chemosensory and ventilatory responses to hypoxia and the hypertension induced by $\mathrm{CIH}$ in conscious rats. We also tested the effects of antioxidant treatment with ascorbic acid on the $\mathrm{CIH}$-induced increase expression of TNF- $\alpha$ and IL$1 \beta$ in the $C B$ to find out whether the upregulation of these cytokines was downstream of the ROS signalling pathways. As the nucleus of the tractus solitarii (NTS) plays a major role in the integration of baro- and chemosensory signals [23], and because of the fact that $\mathrm{CIH}$ increases the number of c-fospositive neurons in the rat NTS, indicating changes in neuronal activity [24, 25], we also addressed the effects of ibuprofen on the number c-fos-positive neurons in the NTS of rats exposed to $\mathrm{CIH}$.

\section{METHODS}

\section{Animals and exposure to intermittent hypoxia}

Experiments were performed on 40 adult male SpragueDawley rats, initially weighing $200 \mathrm{~g}$. Rats were fed with standard chow diet ad libitum, and kept on a 12-h light/dark schedule $(08.00 \mathrm{~h}-20.00 \mathrm{~h})$. Animals were randomly assigned to $\mathrm{CIH}$ or to sham conditions. Researchers unaware of the identity of the treatment performed the physiological recordings and immunohistochemical studies. The experimental procedures were approved by the Bio-ethical Committee of the Biological Sciences Faculty, P. Universidad Católica de Chile, and were performed according to the National Institutes of Health guide for the care and use of laboratory animals.

Unrestrained, freely moving rats housed in individual chambers were either exposed to hypoxic cycles of $5 \%$ inspired $\mathrm{O}_{2}$ for $20 \mathrm{~s}$, followed by room air for $280 \mathrm{~s}$, applied 12 times per hour, $8 \mathrm{~h} \cdot \mathrm{day}^{-1}$ or were kept under sham condition for 21 days [13]. The $\mathrm{O}_{2}$ level in the chambers was continuously monitored using an oxygen analyser (Ohmeda 5120; BOC Healthcare, Manchester, UK) and the $\mathrm{CO}_{2}$ was maintained at a low level by continuous air extraction. Under the sham conditions, the hypoxic exposure was replaced by means of flushing an equal flow of compressed air into the chambers. The room temperature was kept at $23-25^{\circ} \mathrm{C}$.

\section{Chronic subcutaneous ibuprofen treatment}

2 days before the beginning of $\mathrm{CIH}$ or sham exposures, animals were anaesthetised with $3 \%$ isofluorane in $\mathrm{O}_{2}$, and osmotic minipumps (2ML4; Alzet Scientific Products, Chevy Chase, MD, USA) were implanted subcutaneously on the back. The pumps were filled with $400 \mathrm{mg}$ ibuprofen in $2 \mathrm{~mL} \mathrm{NaCl} 0.9 \%$, to achieve a delivering concentration of $\sim 40 \mathrm{mg} \cdot \mathrm{kg}^{-1} \cdot \mathrm{day}^{-1}$, at a rate of $2.5 \mu \mathrm{L} \cdot \mathrm{h}^{-1}$. Control animals were implanted with pumps containing $\mathrm{NaCl} 0.9 \%$.

\section{Ascorbic acid treatment}

In separate experiments, ascorbic acid $\left(1.25 \mathrm{~g} \cdot \mathrm{L}^{-1}\right)$ was administered through the drinking tap water from the first day of $\mathrm{CIH}$ exposure, as previously described [13]. The water solution was freshly prepared every day, and preserved in dark containers to avoid oxidation.

\section{Recording of physiological responses}

Acute experiments were performed in the morning of the day after the last day of the 21 days of $\mathrm{CIH}$ exposure. Rats were anaesthetised with sodium pentobarbitone $\left(40 \mathrm{mg} \cdot \mathrm{kg}^{-1}\right.$ i.p. $)$, followed by additional doses when necessary to maintain a level of surgical anaesthesia (stage 3 plane 2). Rats were placed in supine position and the body temperature monitored by a rectal probe was maintained at $38.0 \pm 0.5^{\circ} \mathrm{C}$ with a heating pad. The trachea was cannulated for airflow recording, and connected to a pneumotachograph to obtain tidal volume $(V \mathrm{~T})$, respiratory frequency $(f R)$, and minute ventilation $\left(V^{\prime} E\right)$. One femoral artery was cannulated with a polyethylene tube, filled with $50 \mathrm{IU} \cdot \mathrm{mL}^{-1}$ of heparin solution for measuring arterial blood pressure with a transducer (Statham P23; StathamGould, Valley View, OH, USA). Cardiac frequency (fC) was measured from the ECG recordings. Physiological variables were acquired with an analogue-digital system (PowerLAB 8SP; ADInstruments, Bella Vista, Australia) and analysed with the Chart 7.2-Pro software. To assess the effects of $\mathrm{CIH}$ on the reactivity of the peripheral hypoxic chemoreflex, we measured ventilatory responses elicited by several isocapnic levels of oxygen tension $\left(\mathrm{PO}_{2} ; 5-670 \mathrm{mmHg}\right)$, maintained until the response was in a steady state $(\sim 10-20 \mathrm{~s})$.

\section{Recording of carotid body chemosensory discharge}

At the end of the ventilatory physiological recordings, one carotid sinus nerve was dissected and placed on a pair of platinum electrodes, and covered with warm mineral oil. The neural signal was pre-amplified (Grass P511; Grass Instruments, Quincy, MA, USA), filtered ( $30 \mathrm{~Hz}-1 \mathrm{kHz})$ and fed to an electronic spike-amplitude discriminator, allowing the selection of action potentials of given amplitude above the noise to be counted with a frequency meter to measure the frequency of carotid chemosensory discharge, expressed in $\mathrm{Hz}$. Carotid sinus barosensory fibres were eliminated by crushing 
the common carotid artery wall between the carotid sinus and the carotid body. The other carotid sinus nerve was cut to prevent vascular and ventilatory reflexes evoked by the activation of the $\mathrm{CB}$. The chemosensory discharge was measured at several isocapnic levels of $\mathrm{PO}_{2}(\sim 5-670 \mathrm{mmHg})$.

\section{Immunohistochemistry for cytokines in the CB}

Quantitative immunohistochemistry was used to address the relative levels of TNF- $\alpha$ and IL-1 $\beta$ in the CB as previously described [13]. Anaesthetised rats were perfused intracardially with PBS at $\mathrm{pH} 7.4$ for $10 \mathrm{~min}$ followed by buffered $4 \%$ paraformaldehyde (PFA; Sigma-Aldrich, Rockville, MD, USA). The carotid bifurcations with the CBs were dissected and postfixed in the same fixative solutions for $12 \mathrm{~h}$ at $4{ }^{\circ} \mathrm{C}$. Samples were then dehydrated in ethanol, included in paraffin, cut into $5-\mu \mathrm{m}$ sections and mounted on silanised slides. After deparaffinisation, samples were submitted to microwave-based antigen-retrieval protocol $(700 \mathrm{~W}$ for $6 \mathrm{~min}$ in citrate buffer $1 \mathrm{M}, \mathrm{pH}$ 6.0). Samples were incubated with $0.3 \% \mathrm{H}_{2} \mathrm{O}_{2}$ to inhibit endogenous peroxidase and then in normal horse serum blocking solution (Vectastain Elite ABC Kit; Vector Lab). Slides were incubated with specific antibodies overnight at $4{ }^{\circ} \mathrm{C}$ in humidity chambers for detection of TNF- $\alpha$ (sc-1350, 1:20, goat anti-TNF- $\alpha$; Santa Cruz Biotechnology, Santa Cruz, CA, USA) and IL-1 $\beta$ (sc-7884, 1:100, rabbit anti-IL-1 $\beta$; Santa Cruz Biotechnology). After rinsing slides in cold PBS, samples were incubated with secondary antibodies conjugated to biotin followed by a ready-to-use stabilised $\mathrm{ABC}$ reagent (Vectastain Elite ABC Kit; Vector Lab, Burlingame, CA, USA), and revealed at $37^{\circ} \mathrm{C}$ in a dark chamber with $3,3^{\prime}$-diaminobenzidine tetrahydrochloride (DAB; Sigma). To avoid false positives during DAB chromogen quantification, special attention was kept to prevent DAB signal saturation. Samples were counterstained with Harris haematoxylin and mounted with Entellan (Merck, Whitehouse Station, NJ, USA). Photomicrographs of the $\mathrm{CB}$ tissue were taken at $\times 100$ with a CCD-camera coupled to an Olympus CX 31 microscope (Olympus Corp., Tokyo, Japan), digitised and analysed using a colour deconvolution algorithm with the ImageJ software (National Institutes of Health, Bethesda, MD, USA). The positive immunoreactive intensity, averaged from four CB fields $\left(9,200 \mu \mathrm{m}^{2}\right.$ each) was expressed as optical integrated intensity.

\section{Immunohistochemistry for c-fos expression in the NTS}

Anaesthetised rats were perfused through the left ventricle with $4 \%$ PFA in PBS ( $\mathrm{pH} 7.4$ ), and the brains were post-fixed in the same fixative for $2 \mathrm{~h}$ and transferred to $30 \%$ sucrose with $0.02 \%$ sodium azide in PBS until they sank. The brainstems were cut frozen under dry ice in the coronal plane, at $50-\mu \mathrm{m}$ thickness, using a sliding microtome. Three alternate series of sections from each brainstem were obtained. One series was stained with cresyl violet, and the other two were used for immunohistochemistry. Free-floating coronal sections of the rat brainstem including the caudal portion of the NTS were incubated in $0.3 \% \mathrm{H}_{2} \mathrm{O}_{2}$ in PBS for $30 \mathrm{~min}$, rinsed in PBS and transferred to the blocking solution $(0.4 \%$ Triton-X100, $0.02 \%$ sodium azide, 3\% normal goat serum in PBS) for $1 \mathrm{~h}$. Sections were incubated overnight at room temperature with the Fos polyclonal antibody (1:20,000; Ab-5, rabbit polyclonal; Oncogene, Cambridge, MA, USA). Sections were rinsed in PBS for $1 \mathrm{~h}$ before being incubated in the secondary antibody solution (1:1,000, Biotin-SP-conjugated AffiniPure goat antirabbit immunoglobulin G; Jackson Immuno-Research, Philadelphia, PA, USA). After rinsing for $40 \mathrm{~min}$, sections were incubated for $1 \mathrm{~h}$ in Vectastain $\mathrm{ABC}$ Elite kit (Vector Lab, Burlingame, CA, USA), rinsed and incubated in a $0.05 \%$ DAB solution containing $0.003 \% \mathrm{H}_{2} \mathrm{O}_{2}$, and $0.05 \%$ nickel chloride to obtain a dark blue reaction product. Photomicrographs of the coronal sections were taken at $\times 4$ with a CCD-camera coupled to an Olympus CX 31 microscope (Olympus Corp.), digitised and analysed using the ImageJ software (National Institutes of Health). The activation of the caudal NTS region induced by $\mathrm{CIH}$ was assessed by counting c-fos-immunoreactive neurons bilaterally in five coronal sections from each rat.

\section{Statistical data analysis}

Data were expressed as mean \pm SEM. Differences between more than three groups were assessed by one- or two-way ANOVA tests, followed by Newman-Keuls or Bonferroni post hoc comparisons.

\section{RESULTS}

The effect of $\mathrm{CIH}$ on $f \mathrm{R}, V \mathrm{~T}, V^{\prime} \mathrm{E}$, systolic (PS) and diastolic arterial pressure $(P D)$ and $f C$ measured at the beginning of the recordings, while rats breathed spontaneously room air, are summarised in table 1 . Baseline $f \mathrm{R}, V \mathrm{~T}, V^{\prime} \mathrm{E}$ and $f \mathrm{C}$ did not differ between any groups ( $p \geqslant 0.05$, one-way ANOVA). Exposure of rats to $\mathrm{CIH}$ for 21 days increased the mean arterial blood pressure due to a significant increase in both $P S$ and $P D$ as compared with sham rats (fig. 1 and table 1$) \quad(p \leqslant 0.001$ Newman-Keuls test after one-way ANOVA). Ibuprofen treatment for 21 days prevented the $\mathrm{CIH}$-induced hypertension (fig. 1). We found that sham rats treated with ibuprofen showed a decrease in arterial blood pressure (table 1 and fig. 1). Nevertheless, the decrease in arterial blood pressure was not statistically different as related to the sham rats $(p \geqslant 0.05$, Newman-Keuls test after one-way ANOVA).

Rats exposed to $\mathrm{CIH}$ showed higher reflex ventilatory responses to acute hypoxia as compared with sham, $\mathrm{CIH}+\mathrm{ibuprofen}$ and sham+ibuprofen rats (fig. 2$)(p \leqslant 0.001$, Newman-Keuls test

\begin{tabular}{|c|c|c|c|c|}
\hline TABLE 1 & \multicolumn{4}{|c|}{$\begin{array}{l}\text { Basal ventilatory and cardiovascular variables } \\
\text { measure at normoxia }\end{array}$} \\
\hline Variable & Sham & $\mathrm{ClH}$ & Sham+lB & $\mathrm{CIH}+\mathrm{IB}$ \\
\hline Subjects $\mathbf{n}$ & 6 & 8 & 6 & 10 \\
\hline$V_{T} \mathbf{m L} \cdot \mathbf{k g}^{-1}$ & $3.3 \pm 0.6$ & $3.0 \pm 0.3$ & $2.9 \pm 0.4$ & $3.6 \pm 0.2$ \\
\hline fR breaths $\cdot \min ^{-1}$ & $74 \pm 11$ & $84 \pm 7$ & $85 \pm 5$ & $74 \pm 6$ \\
\hline Ps mmHg & $118.6 \pm 3.9$ & $156.9 \pm 3.7^{\#}$ & $108.9 \pm 3.3$ & $125.8 \pm 5.1$ \\
\hline PD $\mathrm{mmHg}$ & $104.3 \pm 3.7$ & $114.6 \pm 2.6^{\bullet}$ & $95.8 \pm 2.9$ & $91.9 \pm 3.6$ \\
\hline fc beats $\min ^{-1}$ & & $390 \pm 17$ & & $401 \pm 32$ \\
\hline \multicolumn{5}{|c|}{$\begin{array}{l}\text { Data are presented as mean } \pm \text { SEM. Sham: control animals; } \mathrm{ClH} \text { : rats exposed to } \\
\text { chronic intermittent hypoxia; sham+IB: control rats that received ibuprofen; } \\
\mathrm{CIH}+\mathrm{IB} \text { : rats treated with ibuprofen during the exposure to chronic intermittent } \\
\text { hypoxia; } V \text { T: tidal volume; } f \mathrm{R} \text { : respiratory frequency; PS: systolic blood pressure; } \\
\text { PD: diastolic blood pressure; } f C \text { : cardiac frequency. }{ }^{\#}: p \leqslant 0.001, ~ \\
\text { Newman-Keuls test after one-way ANOVA. }\end{array}$} \\
\hline
\end{tabular}




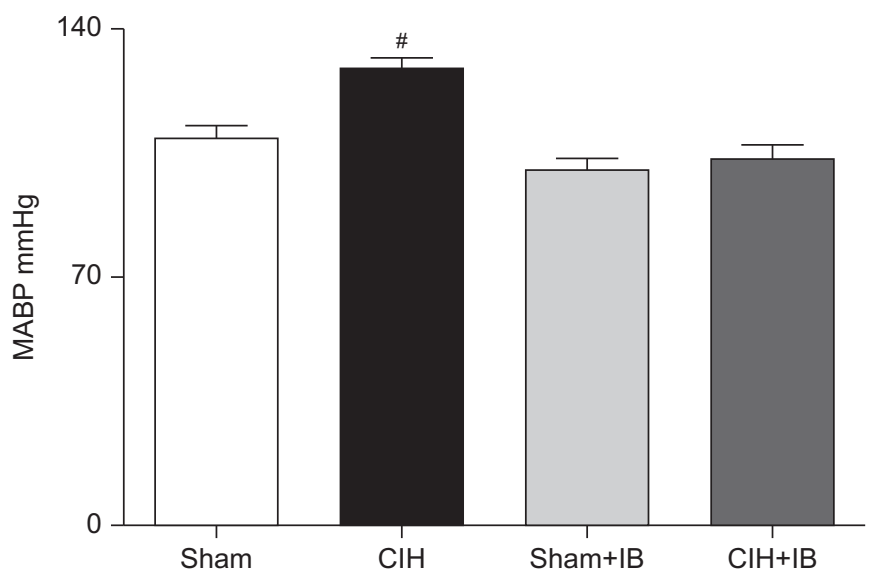

FIGURE 1. Ibuprofen (IB) treatment for 21 days prevented chronic intermittent hypoxia $(\mathrm{ClH})$-induced hypertension in rats. Mean arterial blood pressure (MABP) was measured in six sham rats, eight $\mathrm{ClH}$-treated rats, six sham+IB rats and 10 $\mathrm{ClH}+\mathrm{IB} .{ }^{*}: \mathrm{p} \leqslant 0.001$ compared with sham (Newman-Keuls after one-way ANOVA).

after two-way ANOVA). Thus, treatment with ibuprofen prevented the potentiation of reflex ventilatory responses to acute hypoxia induced by $\mathrm{CIH}$. On the contrary, the $\mathrm{CIH}-$ induced potentiation of the $\mathrm{CB}$ chemosensory response to acute hypoxia was not prevented by ibuprofen (fig. 3). The two-way ANOVA analysis showed that the overall $\mathrm{CB}$ chemosensory curve for $\mathrm{PO}_{2}$ was not different between the $\mathrm{CIH}$ and $\mathrm{CIH}+\mathrm{ibuprofen}$ rats $(\mathrm{p} \geqslant 0.05)$. Ibuprofen treatment did not modify the carotid chemosensory response in the sham rats.

We found significantly increased levels of TNF- $\alpha$ immunoreactivity (TNF- $\alpha$-ir) and IL-1 $\beta$ immunoreactivity (IL-1 $\beta$-ir) in the $\mathrm{CBs}$ from rats exposed to $\mathrm{CIH}$ for 21 days, which was prevented by ibuprofen treatment (fig. $4 \mathrm{a}$ and $\mathrm{b}$ ). As is shown in fig. $4 c$ and $d$, ibuprofen reduced the increased TNF- $\alpha$-ir and

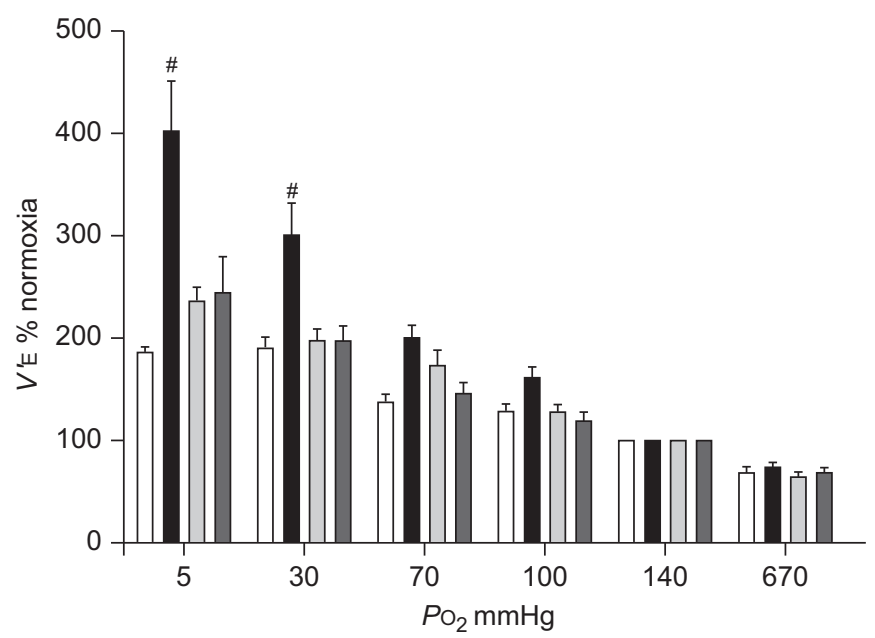

FIGURE 2. Ibuprofen prevented the potentiation of the hypoxic ventilatory response induced by chronic intermittent hypoxia $(\mathrm{ClH})$ exposure. Minute ventilation $\left(V^{\prime} \mathrm{E}\right)$ was measured in response to several levels of inspired oxygen tension $\left(\mathrm{PO}_{2}\right)$ in six sham rats $(\square)$, eight $\mathrm{ClH}$-treated rats $(\square)$, six sham+ibuprofen rats $(\square)$ and 10 $\mathrm{ClH}+$ ibuprofen rats $(\square) .{ }^{\bullet}: \mathrm{p} \leqslant 0.001$ compared with sham, Bonferroni test after twoway ANOVA.

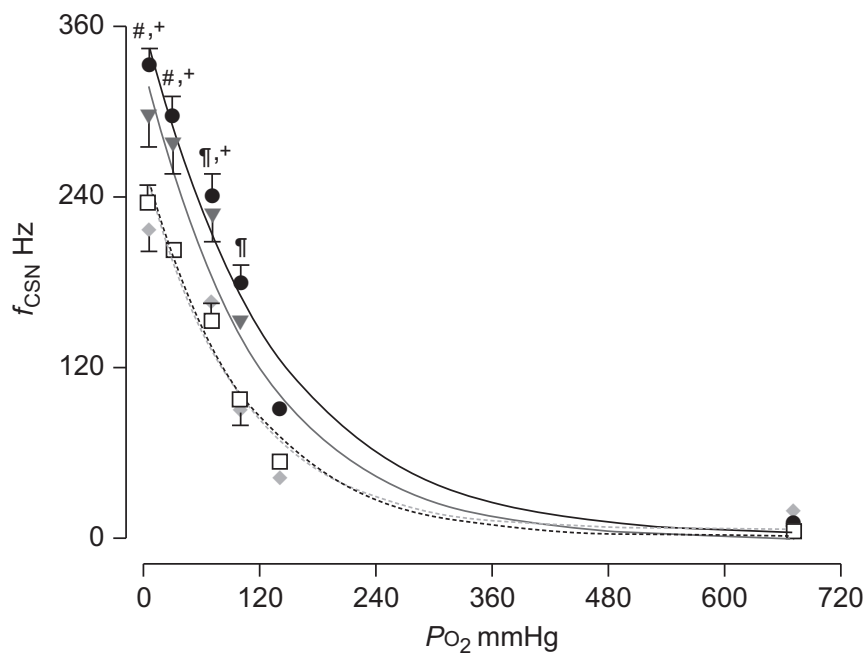

FIGURE 3. Ibuprofen failed to prevent the enhanced carotid body chemosensory responses to hypoxia in rats exposed to chronic intermittent hypoxia $(\mathrm{ClH})$. Summary of the carotid chemosensory responses induced by several levels of inspired oxygen tension $\left(\mathrm{PO}_{2}\right)$ in four sham rats $(\square)$, five $\mathrm{ClH}$-treated rats $(\bullet)$, six sham+ibuprofen rats $(\bullet)$ and $10 \mathrm{ClH}+$ ibuprofen rats $(\mathbf{\nabla})$. fCSN: frequency of chemosensory discharges. ${ }^{\#}: p \leqslant 0.01 ; ": p \leqslant 0.05 ; \mathrm{ClH}$ compared to sham; + : $p \leqslant 0.05 \mathrm{ClH}+$ ibuprofen compared with sham, Bonferroni test after two-way ANOVA.

IL- $1 \beta$-ir by $70 \%$ and $40 \%$, respectively, as compared with the increased optical integrated intensity measured in $\mathrm{CIH}$-treated rats. We did not find differences between the TNF- $\alpha$-ir and IL$1 \beta$-ir levels in the CBs from sham rats treated either with or without ibuprofen $(p \geqslant 0.05)$ (online supplementary fig. S1). The increased levels of TNF- $\alpha$-ir and IL-1 $\beta$-ir in the rat CB exposed to $\mathrm{CIH}$ depended on the oxidative stress, as ascorbic acid treatment during the hypoxia protocol for 21 days reduced the enhanced levels of TNF- $\alpha$-ir and IL- $1 \beta$-ir in the $\mathrm{CB}$ from rats exposed to $\mathrm{CIH}$ (fig. 5). We found that $\mathrm{CIH}$ increased the number of c-fos-positive neurons in the caudal portion of the rat NTS, while ibuprofen treatment attenuated the number of positive neurons (fig. 6). Ibuprofen itself did not change the expression of c-fos. Indeed, we did not find changes in the number of c-fos-positive neurons in the NTS from sham rats treated either with or without ibuprofen $(p \geqslant 0.05)$ (online supplementary fig. S2).

\section{DISCUSSION}

The main findings of this study showed that ibuprofen, which prevented the CIH-increased TNF- $\alpha$ and IL-1 $\beta$ in the CBs and the number of c-fos-positive neurons in the caudal NTS, failed to impede the potentiation of the carotid chemosensory responses to acute hypoxia, but effectively prevented the potentiation of the chemoreflex ventilatory responses to hypoxia as well as hypertension. Thus, the CIH-induced potentiation of the $\mathrm{CB}$ chemosensory responses does not depend on the increased TNF- $\alpha$ and IL- $1 \beta$ levels in the CB, although the increased level of these pro-inflammatory cytokines plays an essential role in the generation of the cardiorespiratory alterations induced by $\mathrm{CIH}$, probably acting at different levels on the hypoxic ventilatory reflex arc and cardiovascular control pathways. In addition, our results showed that ascorbic acid, which prevents the $\mathrm{CIH}$ induced potentiation of the chemosensory responses to hypoxia 
Sham

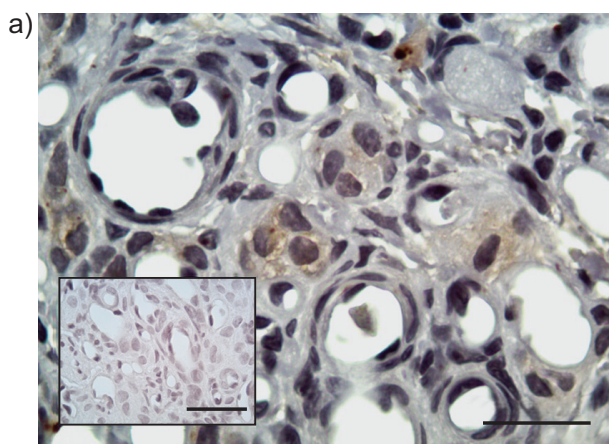

b)

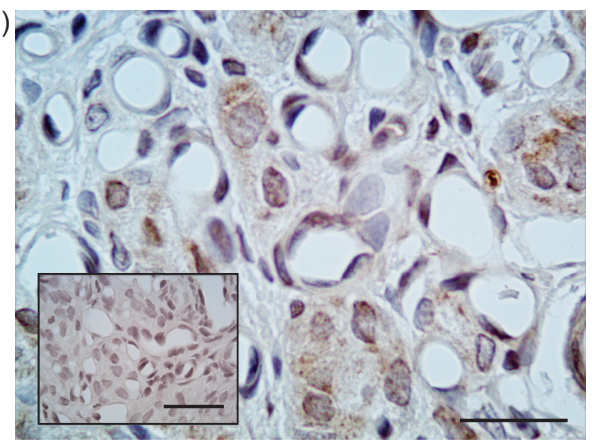

c)

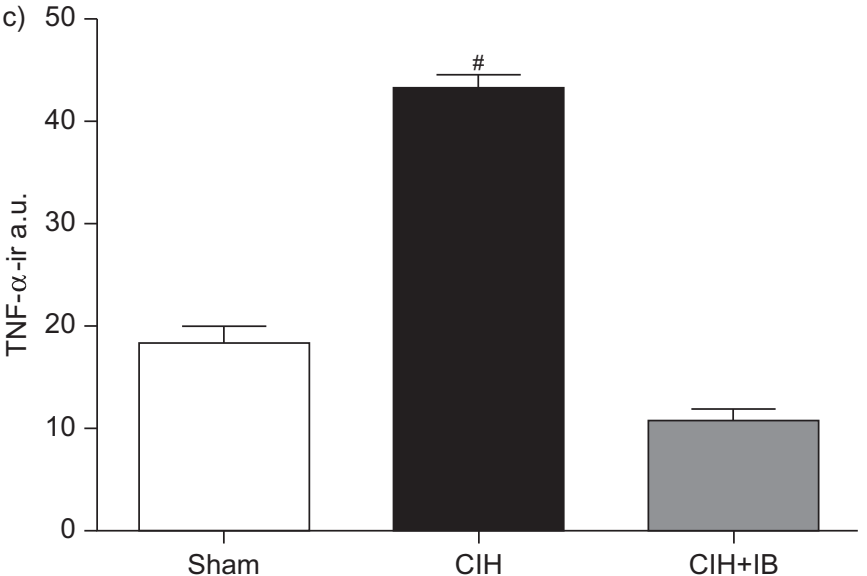

$\mathrm{ClH}$
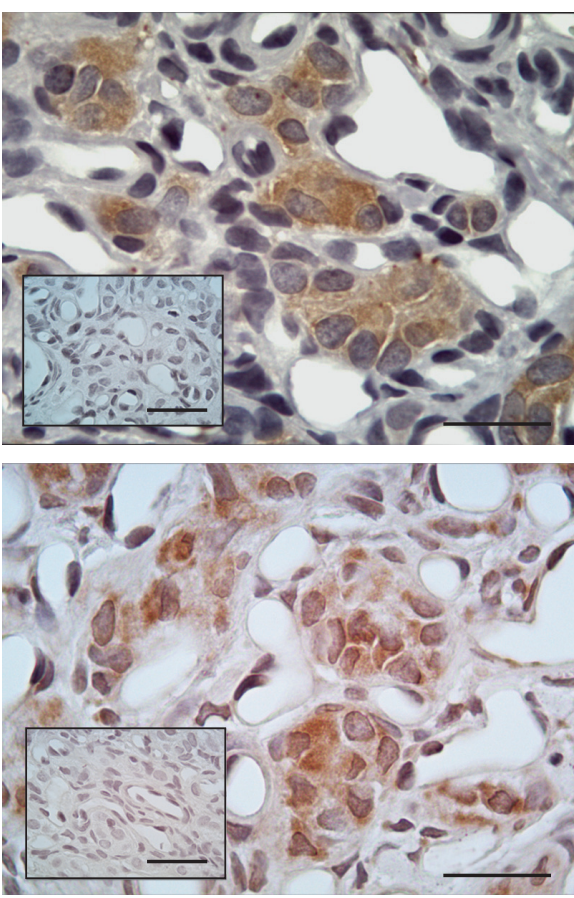
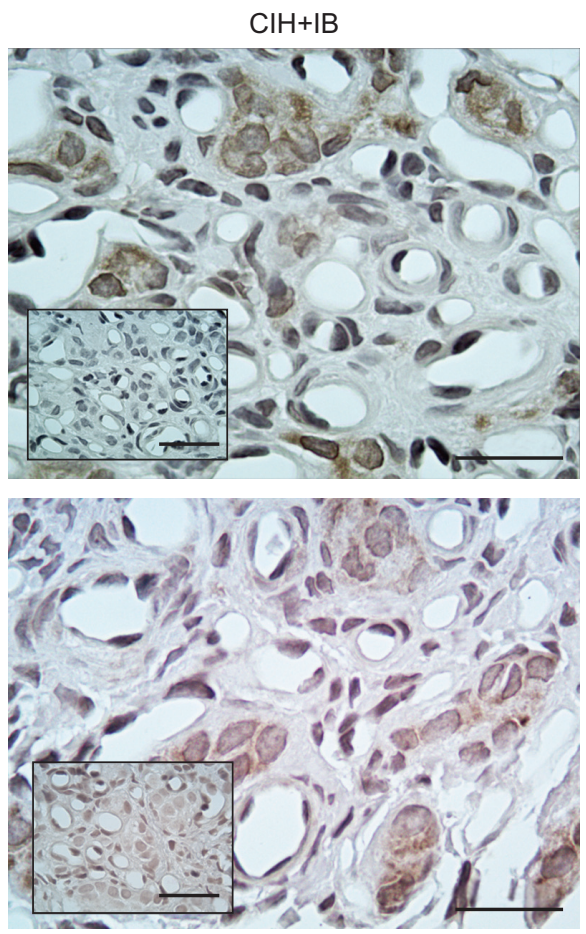

d) 50

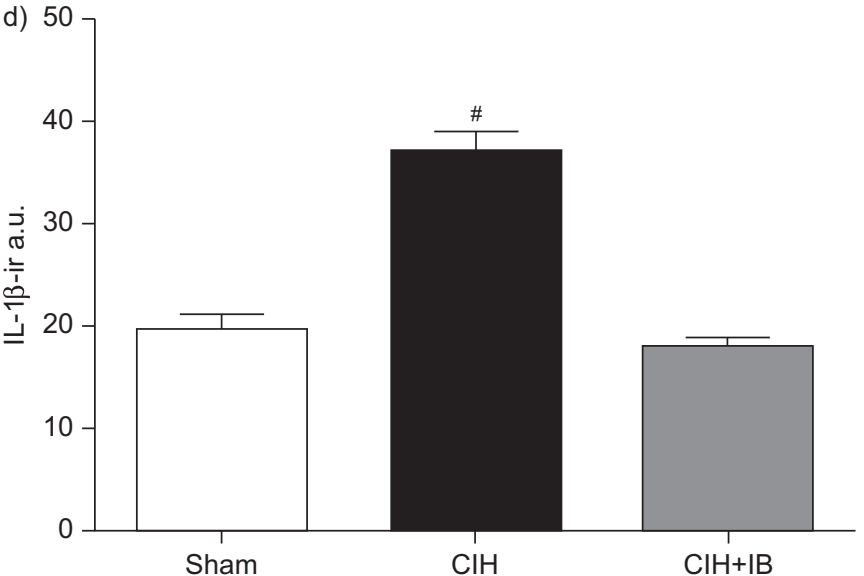

FIGURE 4. Effects of ibuprofen (IB) treatment on the chronic intermittent hypoxia (CIH)-induced increase expression of tumour necrosis factor (TNF)- $\alpha$ and interleukin (IL)-1 $\beta$ in the rat carotid body (CB). Micrographs showing positive immunoreactivity for a) TNF- $\alpha$ and b) IL-1 $\beta$ in CBs from a sham rat, $\mathrm{ClH}$ exposed rat, and CIH rat treated with IB. Insets: negative controls devoid of positive staining. Scale bars $=20 \mu \mathrm{m}$. Quantitative analysis of the positive c) TNF- $\alpha$-immunoreactivity (ir) and d) IL-1 $\beta$-ir measured in the CBs from six sham rats, six $\mathrm{ClH}$ rats and five $\mathrm{ClH}+\mathrm{IB}$ rats. ${ }^{*}: \mathrm{p} \leqslant 0.001$ compared with sham.

and the local oxidative stress in the rat $\mathrm{CB}$ [13], blocked the increased TNF- $\alpha$ and IL- $1 \beta$ in the rat CB, indicating that the increased cytokines levels in the $\mathrm{CB}$ are secondary to oxidative stress. The inhibitory effect of ibuprofen on cytokine accumulation induced by $\mathrm{CIH}$ in the $\mathrm{CB}$ is consistent with its known antiinflammatory effect. Although ibuprofen is considered to be a nonselective inhibitor of cyclo-oxygenases 1 and 2, it is known that ibuprofen inhibits the nuclear translocation of the transcription factor, nuclear factor (NF)- $\kappa \mathrm{B}$, which mediates TNF- $\alpha$ and IL-1 $\beta$ production [26].

\section{Cytokines and CB chemosensory potentiation}

The enhanced production of ROS induced by hypoxicreoxygention cycles evokes the expression of genes and the synthesis of pro-inflammatory cytokines, mediated by the activation of transcription factors such as NF- $\kappa$ B and hypoxia inducible factor $(\mathrm{HIF})-1 \alpha$ [23]. In response to oxidative stress, HIF- $1 \alpha$ induces the expression of several proteins, including ET-1, which transiently increased in rat $\mathrm{CB}$ exposed to $\mathrm{CIH}$ [13]; however, oxidative stress also enhances the expression of pro-inflammatory cytokines, such as IL-1 $\beta$ and TNF- $\alpha$, in the $\mathrm{CB}$, suggesting that chemoreceptor cells can synthesise and release cytokines. Inflammatory processes have been involved in the enhanced reactivity of the $\mathrm{CB}$ chemosensory response to hypoxia in rats exposed to sustained hypoxia [27]. Indeed, LAM et al. [20] found that sustained hypobaric hypoxia recruits macrophages to the rat $\mathrm{CB}$, increases the mRNA expression of IL-1 $\beta$ and TNF- $\alpha$, and interleukin 1 receptor type I (IL-1R1) and 


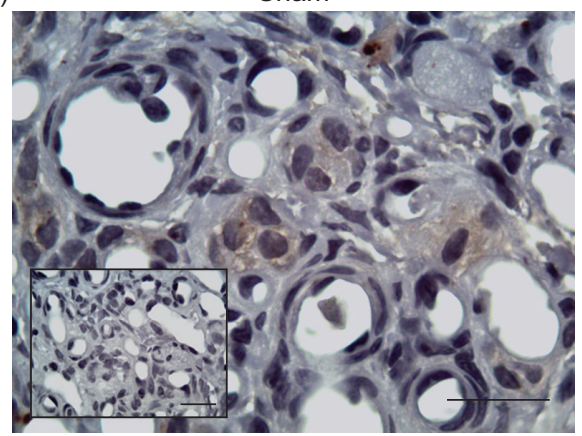

b)

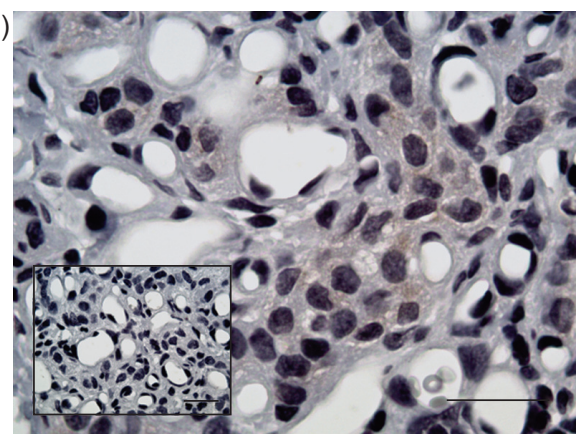

c)

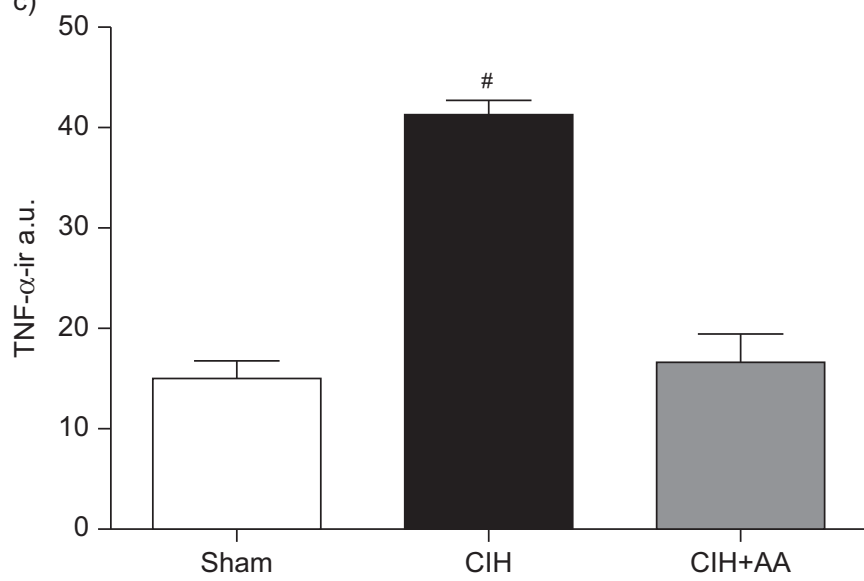

$\mathrm{ClH}$
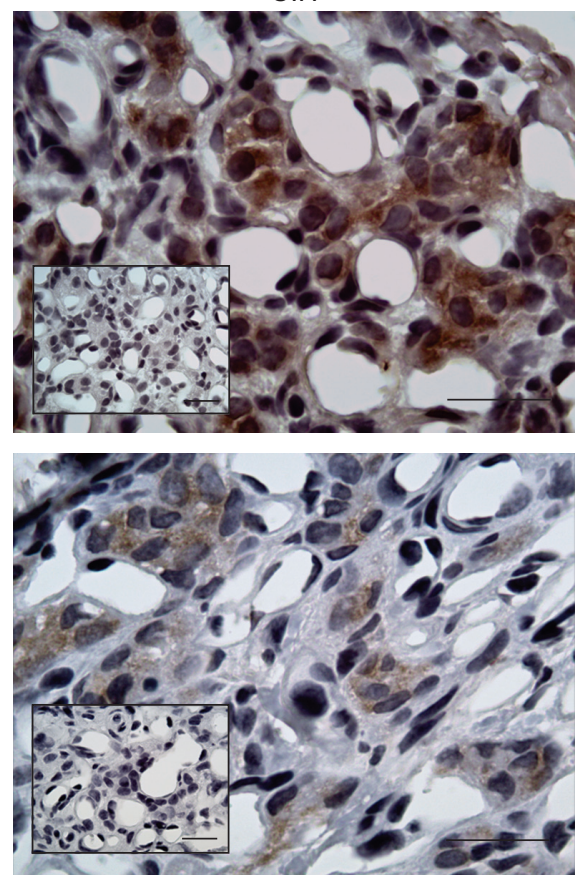

$\mathrm{ClH}+\mathrm{AA}$
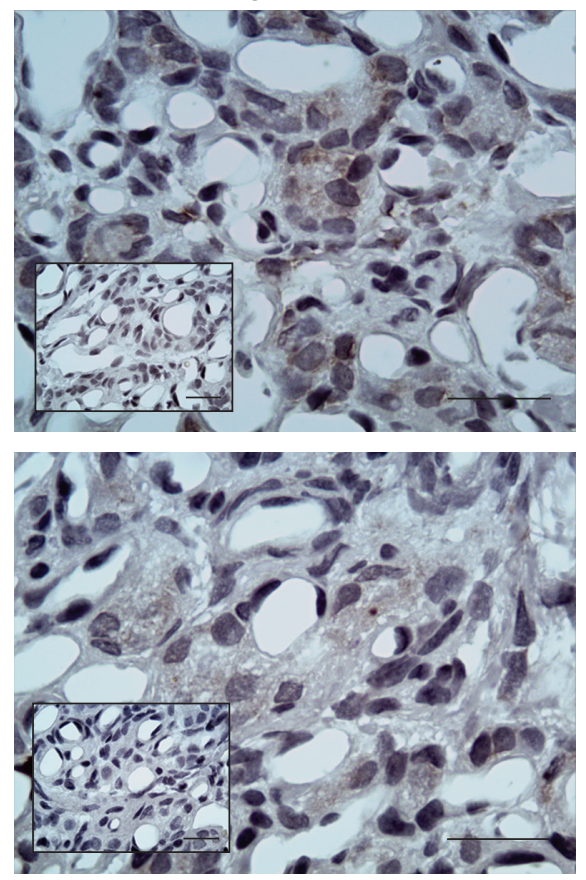

d)

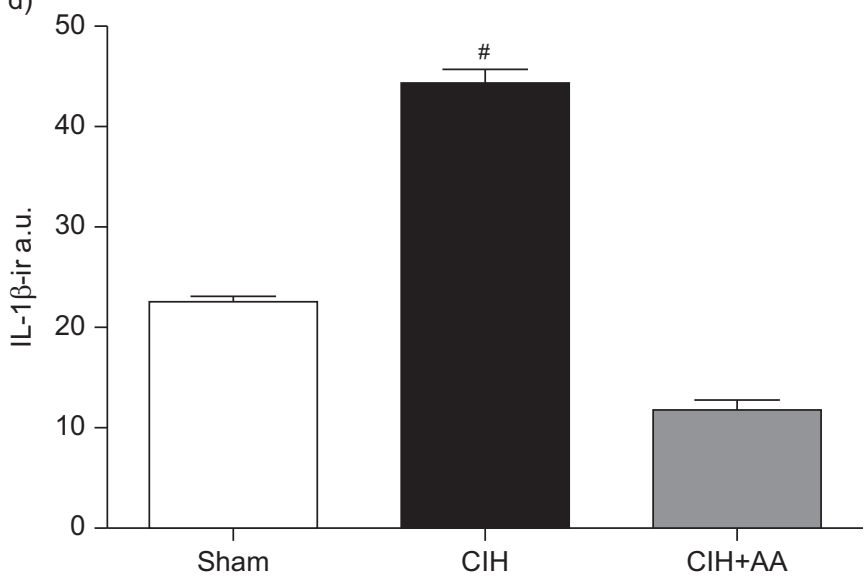

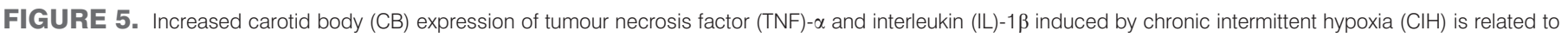

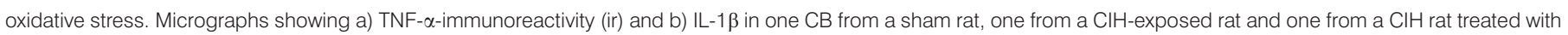

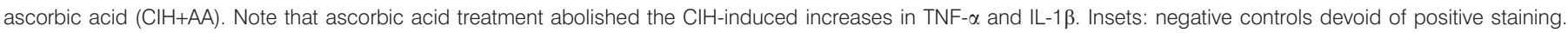

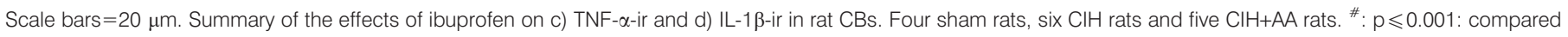
with sham.

tumour necrosis factor receptor type I (TNF-R1) receptors. LIU et al. [21] found that the concurrent administration to rats exposed to sustained hypoxia of ibuprofen and dexamethasone reduced the potentiated $\mathrm{CB}$ chemosensory response to hypoxia, blocked the immune cell invasion and reduced the cytokine RNA expression. Intermittent hypoxia also produces a progressive increase of the TNF- $\alpha$ and IL-1 $\beta$ in the rat CB, but the $\mathrm{CIH}$-induced increases of TNF- $\alpha$ and IL- $1 \beta$-ir was not associated with $\mathrm{CB}$ tissue invasion of immune cell or increased plasma levels, suggesting that $\mathrm{CIH}$ augmented the local production of cytokines in the CB [14]. Present results showed that the $\mathrm{CB}$ chemosensory potentiation to hypoxia induced by
$\mathrm{CIH}$ was not prevented by ibuprofen, while the increased levels of IL-1 $\beta$ and TNF- $\alpha$ in the CB were abolished by the antiinflammatory treatment. Thus, our results suggest that the mechanisms underlying the hypoxic CB chemosensory potentiation induced by sustained and intermittent hypoxia are different.

\section{Cytokines and cardiorespiratory alterations induced by intermittent hypoxia}

The progression of the hypertension in OSA patients and animals exposed to $\mathrm{CIH}$ is associated with increased levels of pro-inflammatory cytokines [1, 2, 5]. Our results strongly suggest that pro-inflammatory cytokines contribute to the 
a)
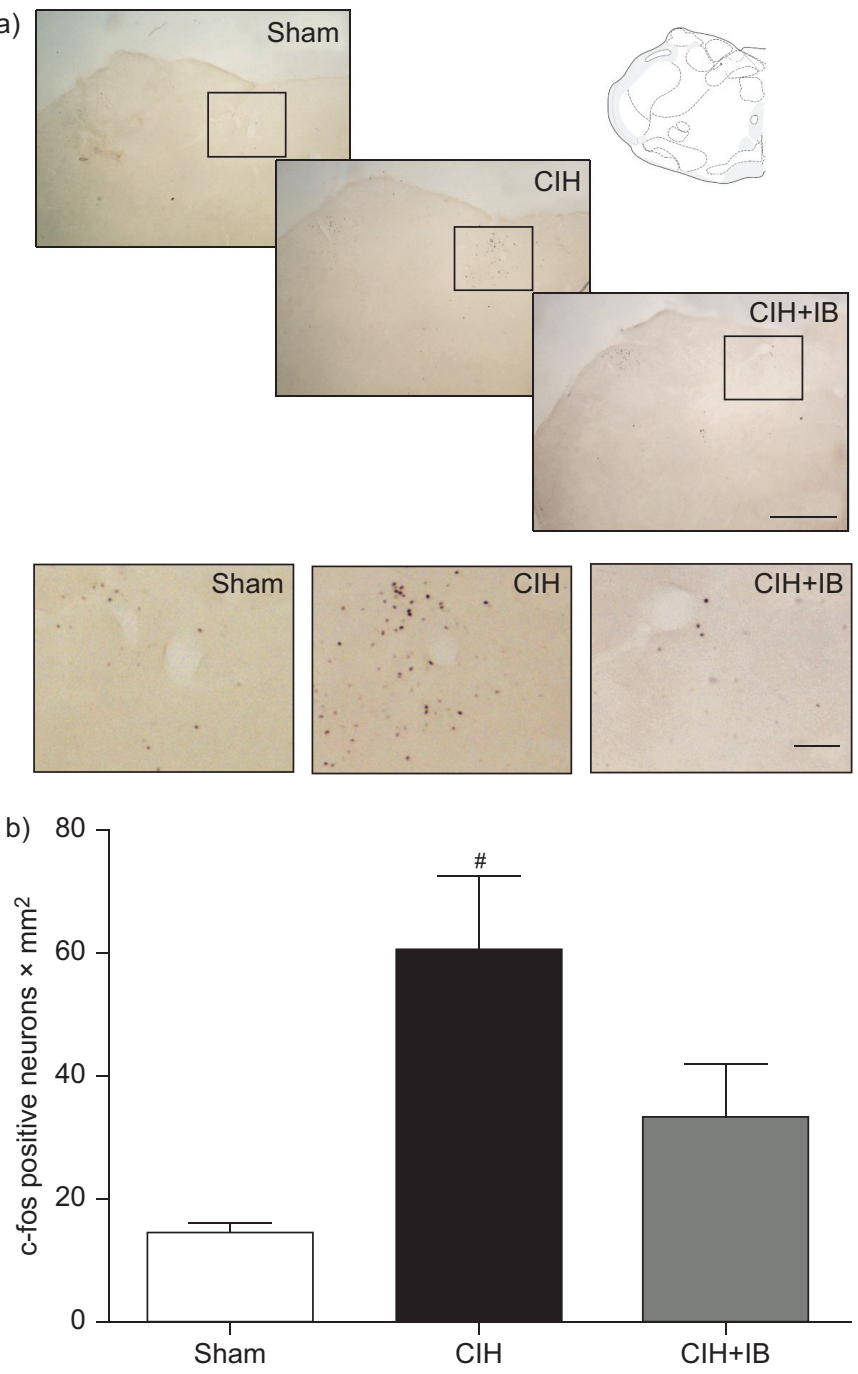

FIGURE 6. Effects of ibuprofen (IB) treatment on the chronic intermittent hypoxia $(\mathrm{CIH})$-induced increases in the number of c-fos-positive neurons in the caudal portion of the rat nucleus tractus solitarii (NTS). a) Representative micrographs showing positive immunoreactivity for c-fos in the NTS from a sham rat, $\mathrm{ClH}$-exposed rat and $\mathrm{ClH}$ rat treated with $\mathrm{IB}(\mathrm{ClH}+\mathrm{IB})$. Low magnification images scale $\mathrm{bar}=600 \mu \mathrm{m}$; high magnification images scale $\mathrm{bar}=200 \mu \mathrm{m}$. Note that IB prevents the increase in c-fos-immunoreactivity in the rat NTS following $\mathrm{ClH}$. b) Summary of the effects of $\mathrm{IB}$. Five sham rats, five $\mathrm{CIH}$ rats and five $\mathrm{CIH}+\mathrm{IB}$ rats. \#: $p \leqslant 0.01$ compared with sham.

$\mathrm{CIH}$-induced cardiorespiratory alterations acting at different levels of the hypoxic chemoreflex and cardiovascular control pathways. PopA et al. [28] reported that ibuprofen treatment blocked the increased ventilatory response to hypoxia and the increased IL-1 and IL-6 protein levels in the brainstem of rats exposed to chronic hypoxia, supporting the proposal that ibuprofen blocks inflammatory processes in the central nervous system (CNS), which contributed to the ventilatory acclimatisation to sustained hypoxia. Present results showed a clear dissociation of the effects of ibuprofen on the $\mathrm{CB}$ potentiated chemosensory responses to hypoxia and to the reflex ventilatory response to hypoxia as well as on the hypertension induced by $\mathrm{CIH}$. Thus, actions of cytokines on the arterial blood pressure and ventilatory reflex responses to hypoxia in rats exposed to $\mathrm{CIH}$ may occur in multiple sites, including the NTS and CNS. Nevertheless, we cannot rule out if the persistent $\mathrm{CB}$ chemosensory potentiation in rats exposed to $\mathrm{CIH}$ and treated with ibuprofen would be detrimental to cardiorespiratory function under long-term exposure to intermittent hypoxia. Future studies addressing the contribution of the $\mathrm{CB}$ chemosensory potentiation to cardiovascular and ventilatory alterations induced by long-term exposures to $\mathrm{CIH}$ are needed.

The available evidence suggests that the cardiorespiratory alterations induced by $\mathrm{CIH}$ originate from the enhanced $\mathrm{CB}$ chemosensory responsiveness to hypoxia [6-9] signals that are transmitted to the NTS, where the respiratory gas and blood pressure sensory signals are primarily integrated. KLINE [23] found evidence that $\mathrm{CIH}$ increased postsynaptic neuron activity in the rat NTS, elicited by an augmented afferent sensory input and enhanced spontaneous synaptic discharge. The idea that CIH increases neural activity in the NTS is also supported by previous studies showing that c-fos immunoreactivity, a marker for neural activation, increased in the rat NTS following CIH $[24,25]$. Moreover, it has been proposed that inflammation in the NTS contributes to the neurogenic hypertension [29, 30]. WAKI et al. [30] found that abnormal gene expression of pro-inflammatory molecules, such as the junctional adhesion molecule 1, are highly expressed in the NTS of spontaneously hypertensive rats and elicit leukocyte accumulation within the vasculature in the NTS. Accordingly, they proposed that cytokines and chemokines might contribute to elevate the arterial pressure by increasing the neuronal activity in the NTS of spontaneous hypertensive rats [30]. Our results, showing that ibuprofen reduced the c-fos immunoreactivity in neurons of the caudal NTS, support a novel role for inflammation in hypertension induced by $\mathrm{CIH}$.

\section{Clinical perspectives}

OSA syndrome is recognised as an independent risk factor for cardiovascular diseases $[1,2]$. Indeed, $\sim 50 \%$ of OSA patients develop diurnal hypertension attributed to oxidative stress and inflammation [1-5]. The gold standard therapy for patients with severe OSA is the application of continuous positive airway pressure (CPAP) during sleep, which reduces the production of ROS and inflammatory molecules and reverses hypertension [1, 2]. However, there are no specific treatments, based on antioxidant or anti-inflammatory drugs, for OSA patients presenting with low adherence to CPAP as well as patients with mild or moderate OSA [1,2]. Thus, a potential therapeutic use of antioxidants and/or anti-inflammatory drugs in OSA deserves further attention. The present results show that ibuprofen treatment prevented the cardiorespiratory alterations induced by $\mathrm{CIH}$, suggesting that anti-inflammatory drugs may potentially be used to ameliorate the hypertension associated with OSA. However, the prolonged use of antiinflammatory drugs, including ibuprofen, may have some risk. Indeed, nonsteroidal anti-inflammatory drugs may increase the risk of cardiovascular thrombotic events, myocardial infarction and stroke, and the risk of gastrointestinal bleeding and ulceration [31]. In addition, prolonged use of ibuprofen may lead to onset of a new hypertension or worsening of a preexisting hypertension [31]. Our results showed that the $\mathrm{CIH}-$ induced overexpression of IL-1 $\beta$ and TNF- $\alpha$ in the CB is 
mediated by ROS and prevented by ascorbic acid, indicating that the increased cytokine levels are secondary to the oxidative stress. Studies performed in rats exposed to $\mathrm{CIH}$ have shown that antioxidant treatment prevents the hypertension [13, 32, 33]. Indeed, we recently reported that $\mathrm{CIH}$ increased plasma lipid peroxidation and 3-nitrotyrosine formation in rat $C B$, enhanced the $C B$ chemosensory and ventilatory responses to hypoxia and produced hypertension [13]. Ascorbic acid treatment prevented the systemic and local CB oxidative stress, the potentiated chemosensory and ventilatory responses to hypoxia as well as the hypertension [13]. Thus, our results support a plausible therapeutic use of antioxidants and anti-inflammatory drugs in OSA patients. Antioxidants seem to be a better choice than anti-inflammatory drugs for the treatment of the OSA-induced hypertension, but anti-inflammatory drugs, such as ibuprofen, used with precaution and in low doses may also be beneficial. Based on the current available information, a long-term combined treatment of antioxidants and anti-inflammatory drugs needs further studies in animal models before being tested in clinical trials.

In summary, the present results suggest that the mechanisms underlying the upregulation of pro-inflammatory cytokines in the $\mathrm{CB}$ induced by $\mathrm{CIH}$ are linked to oxidative stress, as well as the enhanced $\mathrm{CB}$ chemosensory responsiveness to hypoxia. However, the $\mathrm{CIH}$-induced potentiation of $\mathrm{CB}$ chemosensory responses to acute hypoxia does not depend on the increased TNF- $\alpha$ and IL-1 $\beta$ levels. Nevertheless, pro-inflammatory cytokines may contribute to the potentiation of hypoxic ventilatory chemoreflex responses to hypoxia and the progression of the hypertension induced by $\mathrm{CIH}$. This suggests that multiple mechanisms may be involved in the cardiorespiratory alterations induced by $\mathrm{CIH}$.

\section{SUPPORT STATEMENT}

This work was supported by FONDECYT grant 1100405.

\section{STATEMENT OF INTEREST}

None declared.

\section{REFERENCES}

1 Somers VK, White DP, Amin R, et al. Sleep apnea and cardiovascular disease. J Am Coll Cardiol 2008; 52: 686-717.

2 McNicholas WT, Bonsignore MR, the Management Committee of ECAB. Sleep apnoea as an independent risk factor for cardiovascular disease: current evidence, basic mechanisms and research priorities. Eur Respir J 2007; 29: 156-178.

3 Gozal D, Kheirandish-Gozal L. Cardiovascular morbidity in obstructive sleep apnea, oxidative stress, inflammation, and much more. Am J Respir Crit Care Med 2008; 177: 369-375.

4 Lavie L, Lavie P. Molecular mechanisms of cardiovascular disease in OSAHS: the oxidative stress link. Eur Respir J 2009; 33: 14671484.

5 Garvey JF, Taylor CT, McNicholas WT. Cardiovascular disease in obstructive sleep apnoea syndrome: the role of intermittent hypoxia and inflammation. Eur Respir J 2009; 33: 1195-1205.

6 Fletcher EC, Lesske J, Behm R, et al. Carotid chemoreceptors, systemic blood pressure, and chronic episodic hypoxia mimicking sleep apnea. J Appl Physiol 1992; 72: 1978-1984.

7 Narkiewicz K, Van De Borne PJ, Pesek CA, et al. Selective potentiation of peripheral chemoreflex sensitivity in obstructive sleep apnea. Circulation 1999; 99: 1183-1189.
8 Prabhakar NR, Peng YJ, Jacono FJ, et al. Cardiovascular alterations by chronic intermittent hypoxia: importance of carotid body chemoreflexes. Clin Exp Pharmacol Physiol 2005; 32: 447-449.

9 Iturriaga R Moya EA, Del Rio R. Carotid body potentiation induced by intermittent hypoxia: implications for cardiorespiratory changes induced by sleep apnoea. Clin Exp Pharmacol Physiol 2009; 36: 1197-1204.

10 Teppema LJ, Dahan A. The ventilatory response to hypoxia in mammals: mechanisms, measurement, and analysis. Physiol Rev 2010; 90: 675-754.

11 Peng YJ, Prabhakar NR. Reactive oxygen species in the plasticity of respiratory behavior elicited by chronic intermittent hypoxia. J Appl Physiol 2003; 94: 2342-2349.

12 Rey S, Del Rio R, Alcayaga J, et al. Chronic intermittent hypoxia enhances cat chemosensory and ventilatory responses to hypoxia. J Physiol 2004; 560: 577-586.

13 Del Rio R, Moya EA, Iturriaga R. Carotid body and cardiorespiratory alterations in intermittent hypoxia: the oxidative link. Eur Respir J 2010; 36: 143-150.

14 Del Rio R, Moya EA, Iturriaga R. Differential expression of proinflammatory cytokines, endothelin- 1 and nitric oxide synthases in the rat carotid body exposed to intermittent hypoxia. Brain Res 2011; 1395: 74-85.

15 Peng YJ, Nanduri J, Yuan G, et al. NADPH oxidase is required for the sensory plasticity of the carotid body by chronic intermittent hypoxia. J Neurosci 2009; 29: 4903-4910.

16 Osanai S, Mokashi A, Rozanov $\mathrm{CH}$, et al. Potential role of $\mathrm{H}_{2} \mathrm{O}_{2}$ in chemoreception in the cat carotid body. J Auton Nerv Syst 1997; 63: $39-45$.

17 Gonzalez C, Agapito MT, Rocher A, et al. Chemoreception in the context of the general biology of ROS. Respir Physiol Neurobiol 2007; 157: $30-44$.

18 Rey S, Del Rio R, Iturriaga R. Contribution of endothelin-1 to the enhanced carotid body chemoreception induced by intermittent hypoxia. Brain Res 2006; 1086: 152-159.

19 Pawar A, Nanduri J, Yuan G, et al. Reactive oxygen speciesdependent endothelin signaling is required for augmented hypoxic sensory response of the neonatal carotid body by intermittent hypoxia. Am I Physiol Regul Integr Comp Physiol 2009; 296: R735-R742.

20 Lam SY, Tipoe GL, Liong EC, et al. Chronic hypoxia upregulates the expression and function of proinflammatory cytokines in the rat carotid body. Histochem Cell Biol 2008; 130: 549-559.

21 Liu X, He L, Stensaas L, et al. Adaptation to chronic hypoxia involves immune cell invasion and increased expression of inflammatory cytokines in rat carotid body. Am J Physiol Lung Cell Mol Physiol 2009; 296: L158-L166.

22 Semenza GL, Prabhakar NR. HIF-1-dependent respiratory, cardiovascular, and redox responses to chronic intermittent hypoxia. Antioxid Redox Signal 2007; 9: 1391-1396.

23 Kline D. Chronic intermittent hypoxia affects integration of sensory input by neurons in the nucleus tractus solitarii. Respir Physiol Neurobiol 2010; 174: 29-36.

24 Greenberg HE, Sica AL, Scharf SM, et al. Expression of c-fos in the rat brainstem after chronic intermittent hypoxia. Brain Res 1999; 816: 638-645.

25 Sica AL, Greenberg HE, Scharf SM, et al. Immediate-early gene expression in cerebral cortex following exposure to chronicintermittent hypoxia. Brain Res 2000; 870: 204-210.

26 Scheuren N, Bang H, Munster T, et al. Modulation of transcription factor NF- $\mathrm{\kappa B}$ by enantiomers of the nonsteroidal drug ibuprofen. Br J Pharmacol 1998; 123: 645-652.

27 Powell FL. Adaptation to chronic hypoxia involves immune cell invasion and increased expression of inflammatory cytokines in rat carotid body. Am J Physiol Lung Cell Mol Physiol 2009; 296: L156-L157. 
28 Popa D, Fu Z, Go A, et al. Ibuprofen blocks time-dependent increases in hypoxic ventilation in rats. Respir Physiol Neurobiol 2011; 178: 381-386.

29 Paton JF, Waki H. Is neurogenic hypertension related to vascular inflammation of the brainstem? Neurosci Biobehav Rev 2009; 33: 89-94.

30 Waki H, Gouraud SS, Maeda M, et al. Evidence of specific inflammatory condition in nucleus tractus solitarii of spontaneously hypertensive rats. Exp Physiol 2010; 95: 595-600.
31 Rainsford KD. Ibuprofen: pharmacology, efficacy and safety. Inflammopharmacol 2009; 17: 275-342.

32 Troncoso-Brindeiro CM, Da Silva AQ, Allahdadi KJ, et al. Reactive oxygen species contribute to sleep apnea-induced hypertension in rats. Am J Physiol Heart Circ Physiol 2007; 293: H2971-H2976.

33 Kumar GK, Rai V, Sharma SD, et al. Chronic intermittent hypoxia induces hypoxia-evoked catecholamine efflux in adult rat adrenal medulla via oxidative stress. J Physiol 2006; 575: 229-239. 